\title{
I Gesuati e i volgarizzamenti (seconda metà XIV-prima metà XV secolo)
}

\begin{abstract}
I Gesuati formavano non un vero e proprio Ordine, bensì una congregazione animata inizialmente da uomini indotti ma presto aperta a persone in grado di leggere, capire e tradurre dal latino. Il saggio mostra come la presenza di uomini di cultura tra le fila gesuate fosse conseguenza non di un iter istituzionale di istruzione, ma di modalità di reclutamento peculiari. A partire dai primi decenni di esistenza della nuova fondazione, le biblioteche gesuate furono composte in massima parte di volgarizzamenti, realizzati all'interno o all'esterno della congregazione, ma sempre condotti su testi appartenenti alla tradizione monastica riletti alla luce di un ideale apostolico rinnovato, il cui 'spirito' era giusto divulgare anche ai laici e agli indotti.

The Jesuati were not an actual Order, but a congregation, initially driven by uneducated men but quickly welcoming individuals capable of reading and understanding Latin texts and translating them. This essay illustrates that the presence of educated men among the Jesuati did not result from an institutionalized education program, but rather from their specific recruitment practices. In the first decades, the libraries of the Jesuati mainly contained volgarizzamenti which had been produced either within or outside of the congregation. Yet they were always translations of texts stemming from the monastic tradition, reread and rewritten in the light of a new apostolic ideal which was to be conveyed also to the worldly, uneducated public.
\end{abstract}

Parole chiave: Jesuati, Giovanni Colombini, volgarizzamenti

La necessità di volgarizzare, traducendo dal latino per rendere fruibili testi considerati particolarmente sapienti, segna già l'esperienza di Giovanni Colombini, il pio mercante senese attorno al quale si riunì il più antico gruppo di "convertiti" da cui si sarebbe originata la congregazione dei Gesuati. Non ripercorro, in questa sede, il lungo e complesso processo di costituzionalizzazione e, poi, di istituzionalizzazione del movimento religioso promosso da

Isabella Gagliardi, Università degli Studi di Firenze

Ә Open Access. () 2021 Isabella Gagliardi, published by De Gruyter. (cc)BY This work is licensed under the Creative Commons Attribution 4.0 International License. 
Giovanni Colombini e dal suo fraterno amico Francesco di Mino Vincenti a Siena, a metà del Trecento. ${ }^{1}$ In quest'occasione mi limito a ricordare che, per il periodo di nostro interesse, sarebbe errato identificare i Gesuati come membri di un Ordine. La loro struttura di appartenenza fu, piuttosto, un movimento fluido, le cui più antiche Costituzioni datano soltanto al 1425-1426 e sono la risultante di una riflessione comunitaria - all'epoca esistevano già molti degli insediamenti gesuati - avvenuta all'indomani di un'inquisitio ordinaria aperta dall'arcivescovo di Bologna Niccolò Albergati e da quest'ultima condizionata. Quanti facevano parte del movimento pronunciavano i tre voti temporanei di povertà, castità e obbedienza, vestivano un saio bianco, segno dell'approvazione apostolica alla loro forma di vita concessa da Urbano V nel 1367 in forma orale (vivae vocis oraculo), ${ }^{2}$ e conducevano vita in comune pregando e lavorando per sostentarsi ma, non accedendo al sacerdozio, non compivano un cursus studiorum definito. In altre parole i Gesuati erano formalmente indotti, uomini “senza lettere”, cioè privi di una conoscenza puntuale del latino e, perciò appartenenti al cosiddetto "strato culturale intermedio". ${ }^{3}$ Che, poi, il gruppo accogliesse anche persone in grado di leggere, capire e addirittura tradurre dal latino, è vero ed è sicuramente attestato dalla presenza di gesuati volgarizzatori ma è un'altra questione. La loro presenza infatti non era il frutto dell'esistenza di un iter istituzionale di istruzione, bensì era un effetto delle modalità del reclutamento: sono numerosi i notai che seguono l'esempio del Colombini e che decidono di vestire l'abito candido, dono di Urbano V. La loro scelta religiosa è tuttavia conseguenza dei reticolati familiari e amichevoli facenti capo ai seguaci stessi del Colombini, perciò non possiamo ritenere che la conoscenza del latino fosse garantita dall'appartenenza alla congregazione, bensì assumere che accadeva l'esatto contrario.

Dunque, nel caso dei Gesuati, tradurre è di rigore e il volgarizzamento e l'atto di volgarizzare costituiscono un'istanza ineludibile, determinata dall'assetto medesimo del movimento e della congregazione.

1 Me ne sono già occupata in altre sedi: cfr. in particolare Gagliardi, I "Pauperes Yesuati" e Gagliardi, Li trofei della croce.

2 Cfr. Urrutia, “. . . Atque de specifica approbatione Summi Pontificis”.

3 Parafraso la celebre auto definizione di Leonardo da Vinci nel Codice Atlantico («homo sanza lettere») e allo strato culturale dei colti ma che non conoscevano il latino secondo l'etichetta coniata da Carlo Maccagni; per la questione si veda Biffi, Alcune prime osservazioni, pp. 184-185. 


\section{Giovanni Colombini committente - consulente di volgarizzamenti}

Nell'Epistolario di Giovanni Colombini, assemblato dopo la sua morte, trova spazio un interessante scambio epistolare tra lui e un notaio di nome Domenico da Monticchiello che, forse, fu tra coloro che realizzarono l'Epistolario stesso. Domenico era entrato a far parte della brigata de’ povari - così si erano definiti autonomamente gli uomini del gruppo di Colombini e Vincenti - e, con tutta probabilità, i fatti di cui si parla nelle lettere risalgono al 1361-1363. ${ }^{4}$

Colombini aveva chiesto a Domenico di volgarizzare uno scritto importante e complesso: la Mystica Theologia. Composta in ambiente certosino, fu quasi sicuramente opera di Ugo da Balma - priore della Certosa di Meyriat dal 1293 fino all'anno della morte (1305?) - e si era diffusa ben di là delle fila dell'Ordine. ${ }^{5}$ È assai probabile che Giovanni Colombini fosse stato introdotto alla conoscenza della Mystica Theologia dal suo padre spirituale, il certosino Pietro (o Petrone) de' Petroni, di cui avrebbe addirittura composto una Vita, purtroppo perduta. ${ }^{6}$ Domenico era immerso nella traduzione del testo quando scrisse a Giovanni Colombini. Confidò al destinatario e suo maestro di vita spirituale che quella traduzione si era trasformata nell'occasione per comprendere appieno molti fra gli insegnamenti che gli aveva trasmesso oralmente quando si frequentavano. Argomentava: «[. . . ] per le belle cose che io trovava, mi raccendeva spesso e per tal modo, che io non ardiria a scrivare, ma, se piacerà a Cristo, v'el dirò a bocca, e ò intese molte cose, che voi mi diciavate, le quali non

4 Pardi, Sulla vita e sugli scritti, per la datazione delle lettere mi attengo all'ipotesi formulata da Pardi. Su Domenico da Monticchiello si veda il saggio di Levi, Un rimatore senese dedicato all'ultimo periodo della vita del notaio e che ha il pregio di distinguerlo dall'omonimo giurista, cfr. Cellerino, Domenico da Montecchiello.

5 La Teologia Mistica attribuita a San Bonaventura. Sul contenuto dell'opera: Scazzoso, La liturgia-Chiesa dello Pseudo-Dionigi; Scazzoso, I rapporti dello Pseudo-Dionigi; Scazzoso, La teologia antinomica dello Pseudo-Dionigi: I. Per la datazione (tra 1230 e 1290), Dubourg, La date de la Theologia Mystica.

6 L'ultima menzione della Vita composta da Giovanni Colombini e da Niccolò di Mino Vincenti che ho rintracciato sta in una lettera (mutila) indirizzata a Celso Cittadini tra 1610 e 1620 da un anonimo corrispondente (forse Bartolomeo Scala, traduttore della Vita in latino che fu pubblicata negli Acta Sanctorum, Maii 7, Antuerpiae 1688, pp. 188-232). La lettera è conservata a Siena, BCI, Miscellanea D. VII. 11, c. 192r-v. Il 27 gennaio 1611 Celso Cittadini scriveva a Caterina Colombini chiedendole di restituirgli la Vita del Petroni scritta da Colombini che le aveva prestato qualche tempo prima: ibid., c. 164r. Cfr. Gagliardi, I “Pauperes Yesuati”, p. 107, p. 187, p. 501; Gagliardi, Li trofei della croce, p. 8. 
intendeva, né credeva allora». ${ }^{7}$ Si rivolgeva a Giovanni perché era convinto che conoscesse per esperienza diretta ciò che lui stava leggendo nelle carte della Mystica Theologia, e dunque approfittava della sua sapienza carismatica per comprendere meglio il testo e, di conseguenza, tradurlo meglio.

In particolare gli chiedeva lumi intorno a un commento alla lettera di Paolo a Timoteo incastonato da Ugo da Balma nel testo, e che rimaneva particolarmente ostico da capire. Grazie agli scrupoli di Domenico da Monticchiello noi oggi possiamo confrontare il primo abbozzo di traduzione riportato nella lettera a Giovanni e il risultato finale del volgarizzamento, realizzato dopo aver ricevuto la "santa consulenza” del Colombini. L’abbozzo recita:

Le parole [difficili da tradurre n.d.A.] sono queste, secondo che si possono volgarizzare, cioè: ma tu, o Timoteo carissimo, intorno alle mistiche visioni, perfette contrizioni, lassa li sensi tutti e tutte le operazioni intellettuali e ogni cosa sensibile e intellegibile e ogni cosa che è e che non è; e, come t'è possibile, levati su non cognosciutamente alla sua unione, la quale è sopra ogni sustanzia o conoscimento; e da ogni cosa che irretisse, ovvero involgesse, e da ogni cosa assoluta mondanamente, al raggio soprastanziale delle divine tenebre sarai tirato. Vide adunque che neuno di quegli che non sono ammaestrati oda queste cose et coetera.

È molto complicato, se non impossibile, identificare la versione latina utilizzata da Domenico da Monticchiello per il suo volgarizzamento; ciò nonostante non sarà inutile provare a misurare lo sforzo del traduttore recuperando il passo latino che ci interessa. Ricorro a un'edizione seicentesca del testo di Ugo da Balma, dove il brano di cui Domenico scriveva a Giovanni compare in questi termini:

$\mathrm{Tu}$ autem, Timothee amice, circa mysticas visiones forti contritione sensus derelique et intellectuales operationes et omnia sensibilia et intelligibilia et omnia existentia et non existentia, etiam, sicut est possibile, ignote consuerge ad eius Visionem, qui est super omnem substantiam et cognitionem, etenim ecessu tui ipsum, ab omni irretentibili et absoluto medio ad supersubstantialem divinarum tenebrarum radium sursum ageris: Vide autem ut nullus indoctorum ista audiat. ${ }^{8}$

7 Le lettere del B. Giovanni Colombini, p. 40. Ildefonso Tassi precisa: “un’opera uscita dalla certosa da segnalare per il grande influsso da essa esercitato nello sviluppo della metodizzazione della preghiera e della vita spirituale, è la Mistica Theologia [. . .] di Ugo da Balma. Questi fu uno dei primi scrittori metodici sulle vie spirituali; e la sua distinzione divenne l'usuale del linguaggio ascetico: via purgativa, via illuminativa, e via unitiva. Contemporaneo a S. Bonaventura, egli si incontrò nella trattazione delle stesse dottrine col grande dottore mistico", Tassi, Ludovico Barbo, p. 108.

8 Hugonis de Palma Theologia Mystica sive Trivium Sacrum, pp. 46-47. Cfr. anche la versione pubblicata in Patrologia Graeca, 3, coll. 998B-999A (e attribuita a Dionigi l'Areopagita, traduzione di Baltassarre Coderio S.J.). 
La traduzione finale eseguita da Domenico è la seguente:

\begin{abstract}
Ma tu, amico Timoteo, vogliendo tu pervenire all'occulta contemplazione delle cose divine lascia l'uso de' materiali sensi; e simigliantemente la mentale operazione, e tutte quelle cose che si comprendono col senso ovvero con l'intelletto, o vero tutte quelle cose che hanno essere materialmente, ovvero intelligibilmente, siccome è possibile senza cognoscimento rizzati all'unione di colui il quale è sopra ogni sustanzia e cognoscimento intellettuale, per lo levamento di te medesimo sopra te medesimo senza niuna materialità ti leverai ispeditamente e mondanamente su al soprassustanziale raggio delle tenebre divine, il quale è sopra ogni sustanzia. Guarda che niuno degli indotti e non savii non oda queste cose. ${ }^{9}$
\end{abstract}

Come si può evincere dalla lettura dei brani, nella missiva a Giovanni Colombini Domenico annota una traduzione molto letterale, di cui vuol discutere con il suo maestro. Ovviamente trascrive la sua traduzione e non il testo in latino perché Colombini non l'avrebbe compreso. Egli non conosceva quella lingua o, almeno, non la conosceva abbastanza bene da potersi esprimere sul testo originale: è verosimile che "masticasse" soltanto il latino della liturgia e poco altro.

Alla protesta del traduttore sulla difficoltà del brano - «Le quali cose sono forti ad intendere allo 'ntelletto umano», lamentava ${ }^{10}$ - Colombini risponde producendosi in una delle sue abituali lettere infervorate, premettendo tuttavia che gli scrive dopo aver pregato Dio, il che equivale a fornire la più alta forma di legittimazione alle proprie parole. «Però che, come sapete», precisa

Santo Pavolo non disse né scrisse cose maggiori né più alte parole. Tuttavia con grandissimo timore e tremore, confiderommi della bontà dell'Altissimo, e merrò la mano e la penna secondo e come piacerà a lui di porgiare, isperando che alcuno contentamento esso doni alla vostra acciesa e ardente carità. Non volendo però che di questo né di altro che si scriva per me, voi n'abbiate ferma certezza, ma rimanga la verità in sé e ne' veri e savi dichiaratori. Ma tanto vi dico, che la dimanda vostra e lo stato d'essa non è da potere isprimare, né dichiarare, né con penna, né con lingua; ma veramente io mi credo che’l dottore di questo fatto sia sola la potenzia di Dio, e lo scolaio sia solo l'anima, imediati l'uno e l'altro; gli altri ripititori possono più belare che parlare. ${ }^{11}$

L'insistenza sulla purezza della condizione d'ignoranza appare completamente in tema con il contenuto della Mystica Theologia: «l'occulta sapienza dell'amore divino» ${ }^{12}$ che «non puote essere insegnata da uomo, di sotto diremo come

\footnotetext{
9 La Teologia Mistica attribuita a San Bonaventura, p. 78.

10 Le lettere del B. Giovanni Colombini, p. 42.

11 Le lettere del B. Giovanni Colombini, p. 47.

12 Gagliardi, I "Pauperes Yesuati", pp. 33-53; si tratta del manoscritto Collazioni de Santi Padri habitanti nell'erimo di Sciti, citato in Capitoli dei Disciplinati della venerabile compagnia della Madonna sotto le volte dell'I.E.R. Spedale di S. Maria della Scala di Siena, p. 177.
} 
ogni persona quantunque sia laica et idiota, stando nella scuola di Dio ricevarà questa sapienza da lui sopra ogni intelletto per affetto d'amore, la quale niuno filosafo, né niuno maestro, o scolaro secolare, quantunque studii, apprendere nolla puote con intelletto». ${ }^{13} \mathrm{Si}$ tratta di un motivo largamente attestato dalla tradizione cristiana precedente e coeva a Colombini, su cui non mi soffermo.

Domenico, dal canto suo, dopo aver ricevuto la risposta di Giovanni Colombini, gli riscrive entusiasta e gli palesa quali testi aveva compulsato per poter tradurre al meglio l'opera pur senza riuscire a venir a capo del significato di quel passo spiegatogli dal Colombini. Dichiara, infatti: «Però che io ò letto tutto el Vecchio e Nuovo Testamento, Vita e Collazioni de' Santi Padri, quasi tutti gli scritti di Deonisio, el Compendio della Sagra Teologia, la Deosoebia, l'Arlogio della Sapienza, il testi della Mistica Teologia et altri molti libri teologici [. . .]». ${ }^{14}$

Domenico, dunque, volgarizzava “armato” di una buona serie di testi collaterali, gran parte dei quali saranno presenti nelle biblioteche dei Gesuati. In prima istanza occorre identificarli. A parte la Scrittura, aveva letto le Vitas Patrum, forse nel volgarizzamento del Cavalca, le Collazioni de' Santi Padri, forse nella versione presente nella biblioteca della Confraternita della disciplina dell'Ospedale Grande di Siena, cui il più antico gruppo dei povari era legato, ${ }^{15}$ e il Compendio opera, presumibilmente, di Tommaso d'Aquino. ${ }^{16}$ È assai più complicato identificare la Deosoebia. Ad oggi l'unica ipotesi che sono riuscita a formulare è che si tratti del quattordicesimo libro del De Trinitate di Agostino, il cui incipit recita:

Nunc de sapientia nobis est disserendum, non illa dei quae procul dubio deus est (nam sapientia dei filius eius unigenitus dicitur), sed loquemur de hominis sapientia, vera tamen quae secundum deum est et verus ac praecipuus cultus eius est, quae uno nomine theosebeia graece appellatur. Quod nomen nostri sicut iam commemorauimus volentes et ipsi uno nomine interpretari 'pietatem' dixerunt, cum pietas apud graecos eusebeia usitatius nuncupetur, theosebeia vero quia uno verbo perfecte non potest, melius interpretatur duobus ut dicatur potius dei cultus. ${ }^{17}$

Se Deosoebia fosse la corruzione di Theosebeia potremmo supporre che il testo di Agostino fosse stato intitolato così. La lettura del quattordicesimo

13 La Teologia Mistica attribuita a San Bonaventura, p. 32, p. 41. Tra gli esemplari più interessanti del volgarizzamento, si veda il manoscritto conservato in BNCF, Conv. soppr. P. VIII. 1023 (S. Ambrogio), c. 43.

14 Le lettere del B. Giovanni Colombini, p. 52.

15 Gagliardi, I "Pauperes Yesuati”, cap. II.

16 Si veda Tommaso d'Aquino, Compendio di teologia.

17 Opera edita in Patrologia Latina, 42, col. 1055. 
libro del De trinitate sarebbe peraltro coerente agli scopi di Domenico traduttore, perché nelle carte agostiniane si tratta della sapienza. Certamente, se così fosse, doveva trattarsi di un identificativo del testo in uso soltanto nello stretto gruppo dei sodali del Colombini, perché non sono riuscita a reperire nessun'altra attestazione di un simile titolo. L'Arlogio della Sapienza è, evidentemente, l'Horologium Sapientie di Enrico Suso (Heinrich Seuze), un libro che esaltava la philosophia spiritualis, cioè la sapienza sovrannaturale concessa da Dio agli uomini di fede. Suso, del resto, aveva assunto una posizione anti-intellettualista, privilegiando il contatto estatico tra l'anima e il Creatore grazie alla virtù di carità intesa nell'accezione paolina del termine. ${ }^{18}$ Del resto, il volgarizzamento quattrocentesco Oriuolo della sapiença che ho potuto reperire a Firenze, in Biblioteca Riccardiana, ${ }^{19}$ potrebbe essere un codice gesuato, perché la miniatura iniziale che raffigura Suso genuflesso di fronte ad un crocifisso presenta il monogramma gesuato del nome di Cristo apposto sul cuore dell'autore. $^{20}$

I libri usati da Domenico da Monticchiello e, poi, custoditi in copie negli armaria dei conventi gesuati, all'epoca in cui venivano immortalati dalla corrispondenza tra Domenico e Giovanni (a parte il volgarizzamento biblico), sembrano piuttosto complicati per un lettore privo di qualsiasi formazione teologica. È forse ragionevole ipotizzare che, almeno nel caso del testo da volgarizzare (la Mystica Theologia), ci fosse stato l'intervento del monaco certosino Pietro - o, come sembra dopo un accurato studio biografico, Petrone $^{21}$ - della certosa di Maggiano. Si trattava di un esponente dell'aristocratica stirpe dei Petroni: il cardinale Riccardo aveva finanziato la fondazione della certosa di Maggiano e lì aveva preso i voti Petrone, suo parente stretto. A lui Colombini si era legato diventandone un figlio spirituale. Il monaco aveva allacciato numerose relazioni di guida spirituale: è noto il

18 Henrici Susonis Horologium Sapientiae. Per importanti accenni critici all'opera di Suso: Meerssemann, Gli amici spirituali; Guarnieri, Per la fortuna di Ruusbroec; Gennaro, Giovanni Colombini e la sua "brigata", p. 243, nota 1. Infine ai temi cari ai testimoni della devotio moderna di oltralpe rimandano alcune precise affermazioni di Colombini. Ad esempio la seguente «entra tale affetto et amore et affetto d'amore nell'abbisso dell'amore, nelle divine tenebre, divine sono, ch'esso è essa divinità et abbisso; tenebre sono, che sono oscure nel comprenderle e vederle, e più tenebre a parlarne. Questa salita et affogamento impedirebbe ogni considerazione et imaginazione d'alcuna cosa qualunque fosse. Solo l'amore trova l'amore e credo che sia godimento de' godimenti» (Le lettere del B. Giovanni Colombini, p. 50).

19 BR 1782.

20 Sui volgarizzamenti di Suso cfr. anche Bartòla, Per la fortuna di Enrico Suso.

21 Cfr. Petroni, Un documento inedito e anche Pellegrini, Petroni, Pietro. 
fatto che avesse cercato di "convertire" Giovanni Boccaccio, attraverso la mediazione di Francesco Petrarca. ${ }^{22}$

Colombini l'aveva conosciuto frequentando le tornate della Confraternita dei Disciplinati sotto le volte dell'Ospedale di Santa Maria della Scala, dove Petroni era immatricolato fin dall'adolescenza e che, dopo la morte, fu celebrato dai confratelli attraverso un ritratto, collocato sopra la porta di ingresso del loro Oratorio. Colombini, invece, l'onorò scrivendone una Vita in volgare e facendolo a “quattro mani” con Niccolò di Mino Vincenti, il fratello carnale di Francesco Vincenti, amico intimo di Giovanni Colombini e co-istitutore della brigata de' povari. L'originale della Vita si è perso, ma qualcosa dei suoi contenuti è sopravvissuto, perché fu tradotto (e forse rimaneggiato) in latino all'inizio del Seicento da un altro monaco certosino, don Bartolomeo Scala, della certosa di Pontignano presso Siena. ${ }^{23}$ Il ruolo svolto da Petroni nella formazione del Colombini fu sicuramente assai importante: ancora alla fine del Cinquecento il Generale dei Gesuati, Paolo Morigia, era costretto a scrivere che i Gesuati erano stati fondati dal Colombini e non «come hanno detto alcuni, che vogliono che questa religione abbi avuto principio d'un converso dei certosini». ${ }^{24}$ Morigia non rivela l'identità del preteso fondatore, ma non è difficile scorgere dietro quella fake news dell'epoca quantomeno l'eco della figura del Petroni. Peraltro Petroni era monaco, non sacerdote, perché aveva preferito amputarsi uno dei pollici pur di diventare inabile all'officiatura e, per umiltà, sottrarsi all'onore connesso con il percorso e poi la funzione sacerdotale. L'affermazione del Morigia costituisce dunque un segnale indiretto e obliquo sia della solidità del legame tra Petroni e Colombini, sia dell'influenza, percepita dai contemporanei, esercitata dal monaco Petroni sul mercante convertito Colombini.

Così credo che sia stato proprio il monaco Petroni, padre spirituale di Colombini, a consigliargli il testo della Mystica Theologia. Possiamo ragionevolmente supporre che gli abbia parlato di quel libro, che oralmente ne abbia centonato alcune parti e che, di conseguenza, Colombini abbia richiesto a Domenico, colto notaio a lui spiritualmente molto vicino, di tradurre un libro che, evidentemente, considerava sapienziale.

22 Petroni, Un documento inedito.

23 Ibid., p. 140.

24 A testimonianza dell'importante ruolo svolto dai disciplinati e dal Petroni nei confronti della brigata sta un'affermazione polemica di Paolo Morigia: «Questa [da Giovanni Colombini e Francesco di Mino Vincenti] è de' giesuati la vera origine, et non come hanno detto alcuni, che vogliono che questa religione abbi avuto principio d'un converso dei certosini, altri si son messi a scrivere, che questa hebbe cominciamento da una congrega di disciplini, gente mecanica e plebea», Paolo Morigia, Historia dell'origine. 


\section{I frati eredi di Domenico da Monticchiello: i volgarizzamenti gesuati}

L'operazione del tradurre effettuata da Domenico costituì l'incipit di una vera e propria tradizione interna alla congregazione dei gesuati. Nel pieno Quattrocento, mentre era felicemente in corso la costituzionalizzazione del movimento, un gesuato molto colto di nome Antonio Bettini, che fu anche vescovo di Foligno, così descriveva le attività dei confratelli:

Scribunt libros ecclesiasticos, in latino et vulgari sermone et continue commodant libros multos devotos et ecclesiasticos per familias exorando et confortando omnes exemplo et verbis, et cum libris ad contemptum mundi et contemptum vitiorum, exorando et confortando ad virtutes et ad amorem Dei et proximorum. ${ }^{25}$

Bettini, in realtà, enfatizzava la quantità dei testi composti dai gesuati in lingua latina: se è vero che ve ne furono - e del resto lui stesso avrebbe contribuito a scriverli - è altrettanto vero che ambiva a nobilitare la congregazione cui apparteneva egli stesso.

Prima di esporre i risultati delle ricerche sui testi dei Gesuati, occorre premettere che la congregazione sfuggì all'inchiesta sulle biblioteche dei regolari ordinata da Pio V: ${ }^{26}$ usufruendo della protezione accordata dal cardinal Santoro i Gesuati, al pari dei ben più illustri Frati Predicatori e della Compagnia di Gesù, riuscirono a ottenere l'esenzione necessaria. ${ }^{27}$ Pertanto le pagine che seguono sono il frutto di una ricerca personale nei fondi manoscritti di varie Biblioteche italiane. Ciò che sono riuscita a ricostruire è un panorama di massima, non vi è certezza che si tratti di un panorama completo e, anzi, auspico che nuovi ritrovamenti, in futuro, possano completarlo.

Intanto è opportuno sottolineare come la scelta stessa di volgarizzare presupponga un'istanza culturale di base che non va sottovalutata: seppur in tono minore i gesuati si incanalarono nella tradizione che unisce Domenico Cavalca a Iacopo Passavanti, allo scriptorium del convento di Santa Caterina a Pisa e ai

25 BAV, ms. Chigi I. VI. 227, c. $18 \mathrm{v}$.

26 Cfr. il progetto "Ricerca sull'Inchiesta della Congregazione dell'Indice" (RICI) coordinato da Roberto Rusconi e i cui risultati compaiono nella serie "Libri e biblioteche degli Ordini religiosi in Italia alla fine del secolo XVI" della collana "Studi e Testi” della Biblioteca Apostolica Vaticana e i cui volumi più recenti sono La congregazione dell'Indice e Congregazione dei canonici.

27 Sull'intera vicenda, che accomunò i gesuati ad altri Ordini 'minori', si veda il bel saggio di Fragnito, "Dichino corone e rosarii”. 
numerosissimi altri frati, suore e laici che voltarono in volgare testi latini. ${ }^{28} \hat{\mathrm{E}}$ significativo che una cronaca francese del XVII secolo presenti i Gesuati di Tolosa come uomini dotti: «Au mois d'avril de l'an 1425 certains moines qu'on appelait Jésuates ou Clercs Apostoliques, au nombre de cinq vinrent des contrées d'Italie en cette ville. C'étaient des gens de sainte vie et de grande littérature. Par leur institut qui avait été approuvé par la Saint-Siège, ils vivaient du travail de leurs mains». ${ }^{29}$

Nella maggior parte dei casi le traduzioni gesuate rivelano una raffinata sensibilità letteraria: è esemplificativo il prologo preposto ai Dialoghi di san Gregorio da un anonimo volgarizzatore. Le proteste di ignoranza e di inadeguatezza intellettuale sono il topos in cui viene inscritta la scelta del traduttore di privilegiare la fedeltà allo spirito anziché alla lettera del testo, chiarendo che utilizzerà parafrasi e traduzioni non letterali pur di garantire l'intelligibilità dell'opera. Vista la chiarezza con cui l'autore delinea il proprio metodo di lavoro, mi sembra necessario riportare la citazione della fonte per intero:

Però che come dice santo Paulo debitore sono a' savi e a' semplici, perché come religioso di povertà vivo delle lymosina di ciascuno, vedendo non potere essere utile a' savi et licterati per lo mio poco senno e non potere pagare quanto debito a savi perché sono povero di scientia, havevo pensato che ciò che nel conspecto di Dio non fussi al tucto riputato inutile almeno per utilità d'alquanti idioti e non savi di scriptura, recare in volgare il dialogo di sancto Gregorio, il quale infra l'altre opere divote singularmente è utile. Ma volendo cominciare trovai il suo latino in tal modo donato che non mi pare da potere seguitare al tucto l'ordine della lettera, però che tal cosa pare ben donata per gramatica che recata in volgare secondo l'ordine delle parole non pare mettere meno utile. E alcuna volta una piccola parola per grammatica è di tanta significatione che non se ne può trarre intendimento se non per molte parole e volendole recare a volgare intendimento si possono e debbono per trarre più belle sententie molto abreviare. Onde avenga che alquanto mi sia faticoso per lo mio poco intendimento, tucta via il meglio che so e più apertamente reco in volgare il predecto libro; non seguitando a tucto l'ordine delle parole, ma il meglio che posso ponendo la somma e lo intendimento del libro e sforçandomi di seguitare le parole dove convenevolmente posso. E a ciò mi conforta molto santo Gregorio medesimo e induce per suo exemplo, il quale dice infine del suo prolago di questo libro che non intende di seguire l'ordine delle parole a lui donate, ma di dictitare la summa per modo che più piaccia. E così io volendo recare in volgare pongo il volgare più chiaro e più ordinato che posso, e avenga ch'io sia certo che questa opera quanto dalla mia parte biasimar si possa, non ho però voluto lasciare per utilità de' semplici e si avessi trovato alcuno più sufficiente che in ciò si fussi voluto afaticare,

28 Folena, Volgarizzare e tradurre; Delcorno, Predicazione volgare; Severino Polica, Cultura ecclesiastica e cultura subalterna; nonché i volumi Brepols, della serie The Medieval Translator/ Traduire au Moyen Age.

29 Dufner, Geschichte der Jesuaten, p. 369. 
non sarei suto ardito di metterci mano, ma non trovando ò facto il meglio che so, prego adunque gli humili che nne piglino quella utilità che possono, licentia a' savi che la biasimino quante vogliono. ${ }^{30}$

In generale si può sostenere che i testi da tradurre scelti dai gesuati appartenessero alla tradizione monastica. Si tratta di una scelta coerente alla volontà di riforma della congregazione, nelle cui fila il ritorno alla ecclesiae primitivae forma era declinato secondo il paradigma interpretativo che, come ha mostrato Giovanni Miccoli, faceva coincidere i primordi della chiesa con i primordi del monachesimo. ${ }^{31}$ Parallelamente alla diffusione della "lessicografia" spirituale della devotio moderna, la letteratura monastica si accreditava tra i referenti testuali privilegiati per guidare le anime devote. Pur se riassunta, smembrata, centonata, essa costituiva il ricco serbatoio da cui si attingeva per istruire e perfezionare. ${ }^{32}$ I Dialoghi di san Gregorio furono diffusi anche grazie ai Gesuati che, spesso, ne avrebbero impreziosito la veste codicologica per mezzo di miniature che, a loro volta, avrebbero stuzzicato l'immaginazione di artisti e di pittori come, ad esempio, il Maestro del Camposanto pisano o lo Starnina. ${ }^{33}$

I libri volgarizzati (o copiati, qualora fossero già in volgare) erano essenzialmente gli stessi di cui si raccomandava la lettura: le Collazioni di Giovanni Cassiano, ${ }^{34}$ il De consolatione philosophiae di Boezio, ${ }^{35}$ i trattati ascetici di san Bernardo da Chiaravalle o a lui attribuiti, ${ }^{36}$ il già ricordato Horologium Sapientiae di Enrico Suso, ${ }^{37}$ I trenta gradi della scala celestiale e l'esposizione del Pater Noster ancora di Girolamo, ${ }^{38}$ i Moralia (o Moralium libri) di Gregorio

30 BR, ms. 1421 (XV sec), cc. 1r-2r.

31 Cfr. Miccoli, Chiesa gregoriana, pp. 285-389.

32 Cfr. Zarri, La vita religiosa femminile, p. 145.

33 Delcorno, Predicazione volgare, p. 689. Inoltre ricordiamo l'incunabolo Sancti Hieronymi Vita e epistole.

34 BR, ms. 1427 (XV sec.), volgarizzamento anonimo «questo libro è de poveri Ingesuati habitanti a Sancto Giusto delle mura fuor dalla porta a Pinti a Firençe», c. 121v; ms. 1394 (XV sec.). 35 BR, ms. 1597, volgarizzamento anonimo «Finisce el libro de Boetio della philosophica consolatione l'anno del Signiore 1495 a dì .xxv. d'octobre nell'alma città di Firençe. Questo libro è de' frati ingiesuati habitanti a Santo Justo fuori della Porta a Pinti di Firençe, chi lo achatta si ricordi di renderlo per carità» (c. 86r). Peraltro il codice riporta tracce di lettura molto interessanti: si tratta di glosse e note a margine in latino. Altro esemplare, ancora del XV secolo e anonimo BNCF, Pal. 74.

36 BR, ms. 1422 (XV sec.) «Questo libro si è de' poveri di Yesù Christo chiamati Ingesuati a Santa Maria piena di gratia sopra Ligorna», volgarizzamento del De conscientia e del De contemplatione Passionis.

37 BR, ms. 1782 (XV sec.).

38 BR, ms. 1597 (XV sec.) volgarizzamento anonimo; ms. 1422, cc. 103r-132v. 
Magno, la Regula pastoralis ancora di san Gregorio, ${ }^{39}$ i sette salmi penitenziali, ${ }^{40}$ le Esposizioni del Vangelo di Simone da Cascia, i Sermoni, di Bernardo di Chiaravalle, ${ }^{41}$ il Prato spirituale di Giovanni Eucrato, ${ }^{42}$ il Monte dell'orazione ed i Sermoni di sant'Agostino, ${ }^{43}$ i Sermoni di Giovanni Crisostomo, ${ }^{44}$ le Epistole e la Vita di san Girolamo, ${ }^{45}$ la vita dell'abate Isaac di Siria ${ }^{46}$ e numerose altre agiografie. Tra tutte ne ricordo soltanto una presente in un incunabolo, perché la composizione del libro attesta l'intelligenza funzionale di chi lo predisponeva: Sancti Hieronymi Vita e epistole, colla regola del vivere nei monasteri di monache volgarizzata da frate Matteo da Ferrara gesuato, dato alle stampe a Ferrara per i tipi di Lorenzo de' Rossi da Valenza nell'anno 1497.

Nel caso dei Moralia è possibile conoscere i particolari dell'occasione del volgarizzamento. Questo testo, di commento al libro di Giobbe, fu tradotto da Zanobi da Strata fino al capitolo diciottesimo del libro diciannovesimo: Zanobi dunque non completò la traduzione. Giovanni Tavelli comprò un manoscritto dei Moralia il 24 marzo 1438 dal vescovo di Forlì Ludovico Pirani e completò la traduzione. Egli stesso, inoltre, scrive in una sua epistola che il volgarizzamento circolava anche al di fuori dei conventi gesuati. ${ }^{47}$

Si tratta di opere appartenenti al patrimonio cristiano della tradizione, perlopiù, ma con qualche significativa eccezione. Tra gli autori contemporanei spiccano il De disciplina et perfetionis vitae monasticae di Lorenzo Giustiniani tradotto dal Gesuato vescovo di Ferrara Giovanni Tavelli col titolo Trattato della disciplina monastica, ${ }^{48}$ la Quadriga spirituale di Niccolò da

39 Firenze, BML, Acquisti e Doni, 85.

40 BNCF, Pal. 74; BR, ms. 1339 volgarizzati da Giovanni Tavelli da Tossignano, Firenze, BML, cod. 85. Ancora sui volgarizzamenti dei Dialoghi di Gregorio: Dufner, Zwei Werke Gregors des Grossen; Dufner, Die Moralia Gregors des Grossen.

41 BR, ms. 1260 volgarizzati da Giovanni Tavelli da Tossignano. Il volgarizzamento del Tavelli sarà stampato a Venezia nel 1529 dai gesuati di Ferrara, e dedicato a Isabella d'Aragona regina di Napoli. L'edizione più recente a cura di P. Anselmo di San Luigi Gonzaga, I Sermoni di San Bernardo; Angelini, Intorno all'antico volgarizzatore.

42 In questo caso il volgarizzatore fu Feo Belcari: cfr. Il Prato Spirituale de' Santi padri; BR, ms. 1342 (XV sec.).

43 BR, mss. 1354, 1778 e 1318 (XV sec., anonimi). Cfr. anche Wilmart, Formes successives.

44 Siena, BCI, M. VI. 50.

45 BNCF, II. IV. 528 (XV sec.); Siena, BCI, U. III. 13. Di Girolamo, cfr. anche BNCF, Pal. 19, cc. $105 \mathrm{r}-127 \mathrm{v}$ ( $«$ S. Girolamo, sermone dell'assunzione della vergine, mandato a Paola, Eustochio, e a più altre, nel monastero del presepe in Betelem, volgarizzato da un frate dei gesuati di Firenze»).

46 BR, ms. 1354 (XV sec).

47 Con il cuore indiviso, p. 29.

48 Volgarizzamento del Tavelli: BNCF, Conv. soppr. G. VII. 1493 (SS. Annunziata). 
Osimo, l'Ermete Trismegisto (o Pimandro) che, però, non fu volgarizzato dai gesuati ma fedelmente ricopiato dal volgarizzamento effettuato da Tommaso Benci nel 1463 per l'amico Marsilio Ficino. ${ }^{49}$ Feo Belcari, colto amico dei Gesuati, al quale i frati fiorentini avevano concesso la possibilità di “confraternare" con loro, probabilmente aveva volgarizzato anche un altro testo assai importante: I quattro gradi della violenta carità di Riccardo di San Vittore ${ }^{50} \mathrm{e}$ due trattati di Iacopone da Todi. ${ }^{51}$

La veicolazione dei testi così approntati era interna ed esterna alla congregazione.

\section{Il versante interno}

Il già ricordato Paolo Morigia, Generale dei Gesuati e fecondo poligrafo di Milano, per quanto attiene al versante interno, ci narra che i Gesuati dovevano studiare le Costituzioni congregazionali del 1425.

Le Costituzioni erano l'unico testo normativo della congregazione, composto a seguito della già menzionata inquisitio vescovile, ordinata a Bologna dal vescovo Niccolò Albergati a danno della comunità gesuata locale. In quell'occasione i Gesuati di Bologna convocarono una sorta di Capitolo Generale a cui parteciparono tutti i responsabili di tutti gli insediamenti e insieme redassero il testo che raccoglieva gli estremi della loro forma vitae. La trasmissione documentaria delle fonti costituzionali ci ha consegnato questa fonte, cioè l'Ordo et forma morum quos et per consuetudinem observat Congregatio Pauperum qui vulgariter Jesuati nuncupantur. L'esemplare più antico risale al mese di giugno dell'anno 1425 ed è accompagnato da almeno tre volgarizzamenti, di cui uno è di poco successivo al testo latino, risalendo al giugno $1426 .{ }^{52} \mathrm{Il}$

49 BR, ms. 1423 (XV sec.); Garin, La cultura filosofica, p. 152, p. 395.

50 Trattato di Riccardo da S. Vittore de' quattro gradi della carità. La paternità del volgarizzamento è per la prima volta attribuita a Belcari da Moreni, pp. vii-viii.

51 Lettere di Feo Belcari, pp. 55-88. I volgarizzamenti di Iacopone sono traduzioni del Tractatus utilissimus e dei Verba, la cui autorialità non è tuttavia unanimemente condivisa: cfr. Iacopone da Todi, Tractatus utilissimus, Verba.

52 Tra i volgarizzamenti che sono riuscita a rintracciare quello più antico data il mese di giugno dell'anno 1426, quindi fu quasi contemporaneo all'Ordo ed è attualmente conservato in BR, ms. 1792. Qui, a carta 26r si dichiara: «queste cose abbiamo arecchato cossì roççamente in volgare scripto negli anni del Signiore MCCCCXXVI del mese di giugnio». La questione delle fonti normative è documentata e discussa nel mio I "Pauperes Yesuati", al quale rimando. 
capitolo bolognese e le Costituzioni furono un momento fondante nella storia della congregazione, come stanno a testimoniare i giudizi e le considerazioni espresse in merito durante i secoli successivi. I testi erano accompagnati da una Vita di Giovanni Colombini, composta da Giovanni Tavelli da Tossignano, già ricordato. Anche l'agiografia fu volgarizzata e l'esemplare più antico che ho rintracciato fa parte integrante del volgarizzamento delle Constitutiones del 1426. Il confronto con il testo latino ne evidenzia tutta la fedeltà, pur se si nota una certa propensione dell'anonimo traduttore a utilizzare espressioni decise, senza indulgere nelle sfumature e abbreviando molto spesso il disteso periodare latino. Se una simile traduzione da un lato abbassa il livello di eleganza formale del testo, dall'altro ne rafforza l'impatto sull'immaginazione del lettore, colpito dalle numerose frasi colorite, quantunque non particolarmente raffinate. Il volgarizzamento (così come la versione latina della Vita di Colombini) circolò quasi esclusivamente all'interno della congregazione e costituì la fonte principale alla quale attinsero i compositori delle successive Vite di Giovanni Colombini cioè Feo Belcari, Antonio Bettini e Matteo Panici. ${ }^{53}$

Ancora Paolo Morigia scrive che oltre a metà Quattrocento il Gesuato e aristocratico milanese Luca Crivello divenne maestro dei novizi e stabilì che i suoi allievi si sarebbero dovuti «esercitare nello studio dell'opere di Giovanni Cassiano, le quali egli haveva tradotte dal latino in lingua volgare». ${ }^{54}$ Non ho potuto rintracciare il volgarizzamento di Crivelli, perciò possiamo soffermarci soltanto sulla scelta del testo. Si tratta di un libro che forniva gli esempi dei grandi monaci delle origini e sicuramente incentivava in loro la consapevolezza di appartenere a una congregazione che incarnava una forma vitae apostolica e filologica rispetto al cristianesimo primitivo.

Inoltre Morigia precisa che i Gesuati dovevano studiare i Salmi. La conoscenza dei Salmi era di rigore anche perché i Gesuati non accedevano al sacerdozio e dunque dovevano limitarsi alle preghiere per i laici. La cultura che acquisivano stando nella congregazione era, per così dire, quasi da "autodidatta”, cioè conquistata ricorrendo a canali diversi rispetto a quelli canonici. Di fatto al postulante era richiesta soltanto la conoscenza delle nozioni catechetiche fondamentali e gli ignoranti venivano esortati dai superiori ad accontentarsi della «devota rusticitate» riservandosi, tuttavia, di istruirli se avessero

53 Ho pubblicato il volgarizzamento in Appendice a Gagliardi, I "Pauperes Yesuati”, e illustrato e documentato l'intera vicenda della trasmissione della memoria di Colombini in Gagliardi, $L a$ trasmissione della memoria di Giovanni Colombini.

54 Morigia, Paradiso de' Giesuati, p. 321. 
dato prova di umiltà e mitezza. ${ }^{55}$ Secondo Antonio Bettini la composizione dei gesuati era assai variegata: accanto a giovani e promettenti universitari convivevano sacerdoti e religiosi provenienti dal clero regolare, laici esponenti delle fasce sociali più alte e, infine, persone che appartenevano ai ceti più bassi. ${ }^{56}$ Generalmente gli incolti nel Quattrocento restavano a livello di semplici conversi e penitenti o venivano destinati ai romitori superstiti, pertanto dovevano essere in minoranza rispetto alla fetta più consistente della congregazione, stanziata nei conventi cittadini o nei santuari. ${ }^{57}$ Il volgarizzamento delle Constitutiones, in ogni caso, si impose perché era necessario che risultassero comprensibili all'universitas sociorum senza mediazione alcuna, ma soprattutto perché nella congregazione era invalso l'uso di tradurre sistematicamente in volgare gli scritti cui si attribuiva una notevole rilevanza.

Nonostante l'importanza della Costituzioni il testo cardine della vita in comune restava la Santa Scrittura. I Vangeli erano interpretati secondo la lezione agostiniana, riducendo al minimo la glossa: non a caso l'unico volgarizzamento di esegesi neotestamentaria utilizzato dai Gesuati era l'opera di Simone da Cascia. ${ }^{58}$ Nella congregazione fu poi attivo un grande traduttore della Bibbia: Giovanni Tavelli da Tossignano. Egli ne tradusse numerosi libri: Genesi, Esodo, Deuteronomio, I Samuele, Re, Giobbe, Salmi, Proverbi, Ecclesiaste, Cantico dei Cantici, Sapienza, Isaia, Geremia, Ezechiele, Daniele, Osea, Amos, Michea, Abacuc, Matteo, Marco, Luca, Giovanni, Atti, Ep. ai Romani, Ep. I ai Corinti, Ep. II ai Corinti, Ep. ai Galati, Ep. agli Efesini, Ep. ai Filippesi, Ep. ai Colossesi, Ep. I ai Tessalonicesi, Ep. I a Timoteo, Ep. II a Timoteo, Ep. a Tito, Ep. agli Ebrei, Giacomo, Ep. I Pietro, Ep. II Pietro, I Giovanni, Apocalisse. Non tutti i volgarizzamenti furono completati ma è comunque degno di nota lo sforzo effettuato del Tavelli. ${ }^{59}$

55 Ferraresi, Il beato Giovanni Tavelli, I, pp. 101-103. Il Capitolo del 1458 ribadiva: «Item, a dì 2 [maggio] ordinarno et determonarno che chiunque viene alla compagnia che non sapia letera, non gli sia insignato a legere, se prima non è stato nella compagnia tre anni, portandosi bene et reverentemente, ma non portandosi bene né ancho a quello tempo gli debba essere insignato. Sì che passato quello tempo, stia nella discretione del rectore de insegnare o non insegnare a legere o a scrivere, et maxime secondo i portamenti di ciaschuno di quelli che non sanno. Però che se sono superbi et inobedienti non sapendo, molto più serano sapendo». Da questa decisione emerge chiaramente il fatto che i gesuati temevano la cultura in quanto fattore di elevazione sociale e potenzialmente causa di superbia (Dufner, Geschichte der Jesuaten, p. 192).

56 BAV, Chigi, I. VI. 227, c. 6r.

57 Cfr. il mio I Pauperes Yesuati, cap. IV.

58 Cfr. Ferraresi, Il Beato Giovanni, pp. 163-189.

59 Ibid. 
Peraltro le Costituzioni implicitamente obbligavano i Gesuati a assumere il Vangelo quale unica Regola: ${ }^{60}$

questo adunque è il nostro vivere, moderato nel Santo Evangelio di Christo observare, nel quale è la somma d'ogni perfectione, pienissimamente si contiene, al quale non piaccia a Dio che si possa poi pensare che niente di perfectione s'elgli possa agiungnere da uno mortale. E facia che al tecto di questa evengelicha perfectione traendoci l'autore d'esso evangelio con più agievole et più ordinatamente possiamo salire pilgliando prima quelgli tre principali meriti della religione ciò sono povertà, castità e hobedientia. ${ }^{61}$ [. . .] Non però che io dicha queste chosse chossì scripte [ le Costituzioni] sia perfectione di vita, ma abbiamo in esse alchuno principio di bene hoperare et inchominciamento de' converssatione, per le quali andando et referendo di virtù in virtù possiamo pervenire ad l'alta perfectione et alla soma delle virtudi le qualli chopiossamente si truvano, se sarà chi ne cierchi, nello ispatiosso chanpo della Santa Scriptura, il quale si lega et in quella si lavori et non solamente per istudio d'oratione et faticha d'operatione, però che non gli uditori della Parola sua ma i faotori d'essa sarano giustificati. Siché udendo o leggiendo la parolla di Dio et quella guardando e chonservando in chore per memoria et nelle mani per hoperationi noi siamo di quegli invitati però de quali dicie l'apostolo che sempre imparano e mai a scientia di verità non pervengono, ma llevando il cuore ad Dio la munitione del profeta santo il sacrifitio nostro mactutino passi d'olochausto vespertino. ${ }^{62}$

Il valore intrinsecamente normante e costitutivo della vita religiosa che i Gesuati attribuivano alla Scrittura derivava dagli insegnamenti di Agostino e di Girolamo e, in particolare, dal Prologo ad Isaia di san Girolamo, dove i Testamenti erano stati definiti i testi il cui soggetto è il Cristo. La ricezione del magistero di Girolamo, mediato da Agostino, è sicuramente attestata dalla presenza, in ambiente gesuato, di un binomio non scindibile tra i due santi che è ribadito anche dalle rappresentazioni iconografiche di ambiente gesuato, dove Agostino e Girolamo figurano molto spesso in coppia. La congregazione volle intitolarsi a san Girolamo proprio perché vi riconosceva un esempio di umiltà: era il sapiente che aveva abbandonato gli studi per consacrarsi alla traduzione della Scrittura.

Le lettere di Girolamo circolavano tra i Gesuati ed essi le consigliavano ai devoti. All'interno delle miscellanee ascetiche e spirituali dei Gesuati l'epistola che ricorre più spesso è, significativamente, lo scritto incentrato sul valore del-

60 Siena, BCI, K. VII. 27 (Vita et Regula Beati Joannis de Columbinis, 1425), c. 13r: «Erit ergo regula nostra sanctum Christi evangelium observare, in quo omnis perfectionis lumina plenissime continetur cui quicque perfectionis a mortalibus addi posse absit, vel excogitetur a nemine».

61 BR, ms. 1792, c. 2r.

62 Ibid., c. 34 r. 
l'umiltà e indirizzato da Girolamo al senatore sposo di Paola. La lettera di Girolamo, volgarizzata da un Gesuato, così descrive la virtù dell'umiltà:

Humiliato quanto più puoi, che mai non ti potrai aguagliare a l'umiltà di Cristo, però che tu huomo et egli è Dio. Avengha che tu vada schalço et colla tonicha ripeççata e sia come uno de' povari della piaçça et per le case degli enfermi entri per visitarlli et sia occhio de' ciechi e mano de' debili, e piede di çoppi e vada come uno mercenario a portare l'acqua e a speçare la legna et a accendere el fuocho. Tue so' le gotate, ove gli sputi, ove a flagelli, ove la croce, ove la morte che Cristo sostenne. ${ }^{63}$

Le immagini usate da Girolamo vengono assunte e rielaborate dai fratres, ritornando nelle loro composizioni - laudi e scritti ascetici - per qualificare il modello del Gesuato esemplare. Del resto è in nome dell'humilitas che i Gesuati del Quattrocento continuavano a rifiutare gli ordini sacri e avvaloravano la loro decisione allegando, accanto ai testi di Girolamo, la testimonianza di Bernardo da Chiaravalle. Alcuni dicta di Bernardo da Chiaravalle già comparivano nelle lettere del Colombini: molto probabilmente era stato grazie al Petroni che Colombini e i suoi seguaci vi si erano avvicinati ai testi bernardiani. In seguito essi furono adottati dalla congregazione, che li considerò tanto importanti da volgarizzarli, sia quelli apocrifi, sia quelli autentici. ${ }^{64}$

\section{Il versante esterno}

Sappiamo che dopo la morte di Giovanni Colombini, occorsa nel 1367, le sue lettere circolarono nei circoli devoti legati al convento eremitano senese di Sant'Agostino e tra i monasteri femminili che costellavano la Siena di fine secolo, primo fra tutti il monastero delle benedettine intitolato ai Santi Abbondio e Abbondanzio, volgarmente detto di Santa Bonda. Le lettere in uso tra gli agostiniani compaiono copiate insieme ai volgarizzamenti di qualche epistola di Agostino, di trattati a lui attribuiti - I quattro gradi di umiltà, per esempio - e di altra trattatistica ascetica e devota come il De Coscientia apocrifo di Bernardo da Chiaravalle o il trattato Dei dieci stati dell'anima spirituale di Ugo Panziera. ${ }^{65}$

La fortuna quattrocentesca di san Girolamo, delle sue Lettere, delle Vitae Patrum, degli scritti di Cassiano, di Bernardo di Chiaravalle, di Ugo da Balma e di Enrico Suso va riallacciata all'opera di divulgazione dei testi monastici con-

63 Dal volgarizzamento gesuato Siena, BCI, U. III. 13. c. 178r [XV sec.].

64 Sulla ricezione degli scritti e della figura di Girolamo cfr. Rice jr, Saint Jerome in the Renaissance; Russo, Saint Jérôme en Italie.

65 Siena, BCI, Miscellanea, U. III. 16. 
dotta soprattutto dai frati predicatori e dagli eremitani di Sant'Agostino. Gli agostiniani non si applicarono ai volgarizzamenti quanto i frati predicatori, piuttosto centonarono la letteratura monastica nei libelli destinati all'istruzione cristiana e alla direzione di coscienza. ${ }^{66}$

Un altro serbatoio di lettori dei volgarizzamenti Gesuati era costituito dalle monache o, comunque, dalle donne devote. Si tratta di storie difficilmente recuperabili, proprio a causa del complicato assetto istituzionale della congregazione che, fino agli inizi del XVII secolo, non poté intrattenere rapporti formalizzati di cura d'anime perché i suoi componenti non erano sacerdoti. I casi più facili da recuperare sono quelli in cui il volgarizzatore sia anche sacerdote, oltre che Gesuato, perché in quel caso è gli possono essere attribuiti compiti di cura monialium tali da farci comprendere subito chi fossero le destinatarie prime della traduzione. Il già ricordato De disciplina et perfetionis vitae monasticae di Lorenzo Giustiniani fu tradotto dal Gesuato vescovo di Ferrara Giovanni Tavelli col titolo Trattato della disciplina monastica ${ }^{67}$ per le monache di Ferrara e anche il volgarizzamento dei Sermoni di san Bernardo, ancora del Tavelli, tradotto forse per la sorella di Eugenio IV, cioè per Polissena Condulmer, dové avere un qualche successo anche tra le suore, perché la cinquecentina (MDXXVIII) stampata a Venezia «ad instantia de li Frati de li Jesuati di S. Hieronimo» con il titolo Sermoni volgari del divoto Doctore Sancto Bernardo sopra le solennitade di tutto l'anno, nel frontespizio porta una illustrazione in cui Tavelli è accompagnato da due monache genuflesse. ${ }^{68}$ Invece l'edizione veneziana del 1529 del medesimo testo, curata dai Gesuati di Ferrara e dedicata alla regina di Napoli, riporta un frontespizio diverso, in cui Tavelli è ritratto mentre consegna il libro a uno dei due Gesuati che lo accompagnano: segno evidente che la traduzione era usata anche dai frati. ${ }^{69}$ Del resto né i fratres, né le donne erano particolarmente versati nella conoscenza del latino, perciò l'uso volgare era veramente necessario tanto per le une, quanto per gli altri. Inoltre il già menzionato incunabolo Sancti Hieronymi Vita e epistole, colla regola del vivere nei monasteri di monache volgarizzata da frate Matteo da

66 Nella biblioteca del convento di Sant'Agostino di Siena, secondo quanto emerge dall'inventario del 1360, incontriamo le opere di Agostino, Bernardo, Girolamo, Cassiano, Gregorio, etc.: Gutierrez, De antiquis Ordinis Eremitarum, in particolare pp. 301-310. Gli agostiniani senesi non volgarizzarono i testi della tradizione monastica, ma li centonarono per istruire i loro diretti e le loro dirette: Siena, BCI, G. XI. 46, codice del XV secolo dove sono raccolti vari scritti di direzione spirituale di un anonimo agostiniano del XIV-XV secolo e sul quale sto attualmente lavorando per la miscellanea Mendikantische Genderdiskurse, di prossima pubblicazione.

67 Cfr. supra, nota 48.

68 La descrizione del frontespizio in Argelati, Biblioteca degli volgarizzatori, p. 241.

69 Con il cuore indiviso, p. 30. 
Ferrara gesuato, dato alle stampe a Ferrara nel 1497 è un volgarizzamento approntato per una comunità di suore. ${ }^{70}$

Talora erano i legami personali tra Gesuati e sorores a originare, se non proprio un volgarizzamento, almeno la diffusione di un testo tradotto dai Gesuati. Un caso del genere è forse ravvisabile, per quanto in controluce, nel rapporto di paternità - anche spirituale, oltre che naturale - tra Feo Belcari e la figlia Orsola, monaca del monastero femminile di Santa Brigida del Paradiso a Firenze. $^{71}$ Il monastero possedeva il volgarizzamento della Mystica Theologia opera di Domenico da Monticchiello e, forse, era stato proprio il Belcari a fungere da tramite fra le monache e il testo.

\section{Conclusioni}

I Gesuati, in definitiva, parteciparono pienamente al processo culturale e storico dei volgarizzamenti. Intermedi tra laicato e religiosi - tanto che la loro aporia istituzionale produsse, sotto il versante giuridico, interessanti soluzioni che attestano chiaramente il crossing over tra teologia e diritto ${ }^{72}$ - aggiunsero un ulteriore livello allo spazio della negoziazione dell'ordine sociale generato dal processo stesso del tradurre. ${ }^{73}$ Mossi dall'intento di divulgare lo "spirito" e la memoria della chiesa primitiva, nella quale i vertici della congregazione iscrissero fermamente lo stile di vita gesuato rivendicandone l'apostolicità a prezzo di dure lotte interne, essi contribuirono non poco a testimoniare come una riforma della chiesa e della società fosse possibile. La riforma che la congregazione propugnava era semplice: bastava vivere apostolicamente, ovvero lavorando, pregando, mantenendo vivo l'amore per Cristo. Dell'antichità apostolica si fecero vettori anche attraverso la letteratura devota che tradussero e divulgarono, attraverso le Bibbie volgari la cui lettura consigliarono a chi li frequentasse e soprattutto attraverso la pratica quotidiana di una forma vitae al confine con l'affermazione del sacerdozio universale.

$70 \mathrm{Su}$ questo incunabolo cfr. anche l'introduzione del curatore in Epistola di S. Girolamo ad Eustochio.

71 Si veda il mio Circolazione di scritti edificanti. Preciso tuttavia che l'uso di "paternità spirituale" nel testo non ha affatto valore tecnico: mi riferisco al fatto che Feo dispensa consigli spirituali alla figlia, come testimonia l'epistola del 19 ottobre 1454, Lettere di Feo Belcari, pp. 2-16.

72 Il mio I "Pauperes Yesuati" ripercorre il fenomeno dai primordi tardo trecenteschi alla soppressione (1668).

73 Cfr. Pursuing a New Order, I: Religious Education. 
